\title{
Supplemental bifid triple viable capsule treatment improves inflammatory response and $T$ cell frequency in ulcerative colitis patients
}

Shuying $\mathrm{Li}^{1+}$, Yan Yin ${ }^{2 \dagger}$, Dan Xiao ${ }^{3^{*}}$ and Yong Zou ${ }^{4^{*}}$

\begin{abstract}
Background: Ulcerative colitis is a common non-specific chronic disease. Supplementing probiotics has become an important method for the treatment of ulcerative colitis. This study aimed to explore the effect of supplementing bifid triple viable capsules on background mesalazine plus somatostatin on plasma inflammatory factors and $T$ cell frequency in ulcerative colitis patients.

Methods: A total of 130 ulcerative colitis patients admitted to our hospital from August 2018 to March 2020 were included and divided into the experimental group (65 patients with mesalazine plus somatostatin and bifid triple viable capsules for treatment) and the control group (65 patients treated with mesalazine plus somatostatin) using the random number table method. Bifid triple viable bacteria capsules were given orally, $420 \mathrm{mg}$ each time, with 3 times a day for 2 months.

Results: Before treatment, the plasma levels of IL-6, IL-8, hs-CRP, TNF-a, D-lactic acid, and endotoxin (ET), CD4+, CD8+, CD4/CD8 ratio, diamine oxidase (DA0), emotional ability, social ability, intestinal and systemic symptoms were not significantly different between the two groups (all $P>0.05$ ). After treatment, the plasma levels of IL-6, IL-8, hs-CRP, and TNF-a decreased in both groups, and were lower in the experimental group than those in the control group (all $P<0.05)$. The levels of CD4+ and CD4/CD8 ratio increased, and were higher in the experimental group than those in the control group $(P<0.05)$; the CD8+ levels were reduced, and were lower in the experimental group than those in the control group $(P<0.05)$. The plasma D-lactic acid, ET, and DAO levels were decreased, and were lower in the experimental group than those in the control group; emotional ability, social ability, intestinal and systemic symptoms were improved, and were higher in the experimental group than those in the control group (all $P<0.05$ ). During the course of treatment, 2 cases of abdominal discomfort and 1 case of rash occurred in the experimental group, with an adverse event rate of $4.62 \%$ (3/65); 3 cases of abdominal discomfort and 2 cases of rash occurred in the control group, with an adverse event rate of $7.69 \%(5 / 65)$.
\end{abstract}

\footnotetext{
*Correspondence: 8592209@qq.com; 83807973@qq.com

${ }^{\dagger}$ These authors contributed equally to this work: Shuying Li and Yan Yin.

${ }^{3}$ Department of Gastroenterology, The Sixth Hospital of Wuhan, Affiliated

Hospital of Jianghan University, No.168 Hongkong Road, Wuhan 430015,

China

${ }^{4}$ Emergency Department, Wuhan Emergency Medical Center, No.288

Machang Road, Jianghan District, Wuhan 430022, China

Full list of author information is available at the end of the article
}

(C) The Author(s) 2021. Open Access This article is licensed under a Creative Commons Attribution 4.0 International License, which permits use, sharing, adaptation, distribution and reproduction in any medium or format, as long as you give appropriate credit to the original author(s) and the source, provide a link to the Creative Commons licence, and indicate if changes were made. The images or other third party material in this article are included in the article's Creative Commons licence, unless indicated otherwise in a credit line to the material. If material is not included in the article's Creative Commons licence and your intended use is not permitted by statutory regulation or exceeds the permitted use, you will need to obtain permission directly from the copyright holder. To view a copy of this licence, visit http://creativecommons.org/licenses/by/4.0/. The Creative Commons Public Domain Dedication waiver (http://creativeco mmons.org/publicdomain/zero/1.0/) applies to the data made available in this article, unless otherwise stated in a credit line to the data. 
Conclusion: The supplementary treatment of bifid triple viable capsules can effectively enhance the curative effect in ulcerative colitis patients, reduce plasma inflammatory factors, and regulate T cell frequency, which is worthy of clinical application.

Keywords: Mesalazine, Somatostatin, Bifid triple viable capsules, Ulcerative colitis, Plasma inflammatory factors, T cell frequency

\section{Background}

Ulcerative colitis is a common non-specific chronic disease, which can involve the large intestine mucosa and submucosa, with abdominal pain, mucus pus and blood in the stool, and diarrhea as the main clinical manifestations [1]. It has a protracted course, is extremely difficult to treat and recurs easily, which has a serious impact on the patient's normal life and work. Therefore, selecting an appropriate treatment regimen is a key clinical issue that needs to be resolved urgently. Recent studies have confirmed that the main characteristic of ulcerative colitis is that the intestinal wall is infiltrated and continuously activated by a large number of inflammatory cells, for which the occurrence and development are closely related to a variety of inflammatory factors such as interleukin-6 (IL6), interleukin-8 (IL-8), high-sensitivity C-reactive protein (hs-CRP) and tumor necrosis factor (TNF)- $\alpha$ [2]. Currently, there are few clinically effective methods for the treatment of ulcerative colitis. Antibacterial agents, glucocorticoids, steroid hormones and aminosalicylic acid preparations are commonly used, in which the first choice is the aminosalicylic acid preparations [3].

With the deepening of research and the development of the medical levels, it has been found that ulcerative colitis is closely related to an imbalance of the intestinal flora [4]. The addition of probiotics during treatment has become a novel treatment concept and method [5]. Probiotics have a promoting effect on maintaining the balance of the flora in the intestinal tract and can be used for the treatment of a variety of intestinal diseases, and play a good auxiliary synergistic effect [6]. The composition and proportion of the intestinal flora play an important role in the occurrence and progression of ulcerative colitis [7]. When the intestinal flora is disturbed, the food residues in the intestine are abnormally fermented under the action of harmful bacteria to produce detrimental substances, causing damage to the intestinal cavity and mucous membranes. As a result, patients may develop abdominal pain, diarrhea, hematochezia and other symptoms, resulting in the occurrence of ulcerative colitis or aggravation of the disease [8]. Bifid triple viable capsules can promote local granulation growth and vascular remodeling of the intestinal ulcer tissues and accelerate rapid healing of the ulcer surface. In addition, bifid triple viable capsules can increase the content of probiotics, improve the barrier function and immune function of the intestinal tract, thereby reducing the inflammatory response. Research conducted by Zhang et al. [9, 10] confirmed that the bifid triple viable bacteria can produce lactic acid and acetic acid after entering the body, reduce the $\mathrm{pH}$ of the intestinal lesions, inhibit the reproduction of harmful bacteria, and increase the proportion of intestinal probiotics by adjusting the proportion of intestinal flora, and restore the normal balance of the intestinal flora of the body. Somatostatin is a peptide hormone with 14 amino acids isolated from the hypothalamus, which is a neuropeptide with a variety of physiological functions [11]. It can inhibit large amounts of vasodilators such as vasoactive intestinal peptides and the secretion of cellular inflammatory factors such as interleukins, reduce hepatic artery blood flow and intrahepatic vascular resistance, protect gastrointestinal mucosa, and relieve inflammation symptoms [10]. Somatostatin has a certain protective effect on ulcerative colitis patients, and it also has a variety of regulatory effects on the immune system [11]. Mesalazine has the function of inhibiting colonic mucosa secretion [12].

However, there are few clinical reports on the treatment of ulcerative colitis with mesalazine plus somatostatin and bifid triple viable capsules. Therefore, this study enrolled 130 ulcerative colitis patients admitted to our hospital and explored the effect of mesalazine plus somatostatin and bifid triple viable capsules on plasma inflammatory factors and $\mathrm{T}$ cell frequency in ulcerative colitis patients, thereby providing a basis for the treatment of ulcerative colitis.

\section{Methods}

\section{Clinical data}

A total of 130 ulcerative colitis patients who were admitted to our hospital from August 2018 to March 2020 were included and divided into the experimental group and the control group using the random number table method. There were 65 cases in each group. The study group were 27-55 years old, with a mean age of $(40.83 \pm 3.21)$ years, and had 33 males and 32 females; the control group were $28-54$ years old, with a mean age of $(40.79 \pm 3.19)$ years, and had 34 males and 31 females. There was no statistically significant difference in general data between the two groups $(P>0.05$, 
Table 1 General data of patients in the two groups

\begin{tabular}{|c|c|c|c|c|}
\hline & $\begin{array}{l}\text { Experimental group } \\
(n=65)\end{array}$ & Control group $(n=65)$ & $x^{2}$ vale & $P$ value \\
\hline Age (years) & $40.83 \pm 3.21$ & $40.79 \pm 3.19$ & 0.071 & 0.944 \\
\hline Male/female & $33 / 32$ & $34 / 31$ & 0.031 & 0.861 \\
\hline $\mathrm{BMI}\left(\mathrm{kg} / \mathrm{m}^{2}\right)$ & $23.67 \pm 0.21$ & $23.66 \pm 0.19$ & 0.285 & 0.776 \\
\hline Course of disease (h) & $24.36 \pm 2.08$ & $24.21 \pm 1.76$ & 0.444 & 0.658 \\
\hline \multicolumn{5}{|l|}{ Degree of disease $(n)$} \\
\hline Mild & 19 & 21 & 0.144 & 0.704 \\
\hline Moderate & 46 & 44 & & \\
\hline \multicolumn{5}{|l|}{ Range of lesions (n) } \\
\hline Left hemi-colon & 17 & 15 & 0.166 & 0.684 \\
\hline Pancolitis & 48 & 50 & & \\
\hline \multicolumn{5}{|c|}{ Pre-treatment mayo score (points) } \\
\hline Mild & $7.21 \pm 0.63$ & $7.13 \pm 0.59$ & 0.415 & 0.680 \\
\hline Moderate & $8.26 \pm 2.29$ & $8.58 \pm 2.13$ & -0.458 & 0.649 \\
\hline
\end{tabular}

BMI, Body Mass Index

Table 1). All patients voluntarily participated in the study and signed an informed consent form, and the study protocol complied with the relevant requirements of the Declaration of Helsinki of the World Medical Association. This study was approved by the Ethics Committee of Affiliated Hospital of Jianghan University.

\section{Inclusion and exclusion criteria}

The inclusion criteria were as follows: patients met the diagnostic criteria for ulcerative colitis in the "Interpretation of Guidelines for the Diagnosis and Drug Treatment of Inflammatory Bowel Disease" [13], with typical clinical symptoms, and were diagnosed as ulcerative colitis by fiber colonoscopy; patients with a clear history and allergy history, complete clinical data; patients had clear consciousness, and stable vital signs; patients with good compliance and normal mental health. Exclusion criteria were as follows: patients had serious primary diseases in systems such as the cardiovascular, liver, kidney and hematopoietic system; patients had organic diseases such as colon cancer, disease of the biliary tract and pancreas, etc.; patients had endocrine and metabolic diseases such as blood coagulation, hematologic diseases, and genetic diseases, etc.; patients had other gastrointestinal diseases (bacterial dysentery, Crohn's disease, and ischemic colitis, etc.); pregnant or lactating women; patients were allergic to the drugs in this study; patients received surgical treatment, radiotherapy and chemotherapy and other drug treatments; patients did not strictly follow the study protocol.

\section{Treatment regimen}

All patients were given nutritional intervention, and were informed to eat more easily digestible foods, to ensure the intake of high-protein substances, and avpod spicy and stimulating foods. Patients in the control group received mesalazine plus somatostatin, in which mesalamine was taken orally [manufacturer: Sunflower Pharmaceutical Group Jiamusi Luling Pharmaceutical Co., Ltd.; Approval Number: National Medicine Standard H19980148], 1.0 g each time, 4 times a day; somatostatin [manufacturer: Swiss Serono Co., Ltd.; Approval Number: Registration Certificate No. X19990113] was dissolved in 5\% glucose solution. It was first intravenously injected with $250 \mathrm{mg}$, and then immediately given intravenous administration at a rate of $250 \mathrm{mg} \cdot \mathrm{h}-1$. Patients in the experimental group received bifid triple viable bacteria capsules in addition to the treatment in the control group. Bifid triple viable bacteria capsules [manufacturer: Shanghai Xinyi Pharmaceutical Co., Ltd.; approval number: National Medicine Standard S10950032] were given orally, $420 \mathrm{mg}$ each time, 3 times a day. The above-mentioned treatments were continued for 2 months.

\section{Observational indicators}

The clinical efficacy and adverse events (AEs) in the two groups of patients were observed after treatment, and the plasma inflammatory factors (IL-6, IL-8, hsCRP, and TNF- $\alpha$ )] and T cell frequency (T lymphocyte subpopulations CD4 and CD8+, and CD4/CD8 ratio), markers for intestinal mucosal barrier function [plasma D-actic acid, plasma endotoxin (ET), and diamine 
oxidase (DA0)] and quality of life (QoL) before and after treatment were compared.

Clinical efficacy: according to the "Consensus Opinions on the Diagnostic and Treatment Standards of Inflammatory Bowel Disease in China" [14], the clinical efficacy was evaluated. Cure: clinical symptoms and signs disappeared; endoscopic colonoscopy showed no obvious abnormalities in the intestinal mucosa, and no recurrence during follow-up; markedly effective: clinical symptoms and signs largely disappeared, and colonoscopy showed mild mucosal inflammation; effective: clinical symptoms and signs were improved, and colonoscopy showed improvement in the intestinal mucosa; invalid: none of the above standards had changed. Total effective rate $=$ effective rate + markedly effective rate + cure rate.

Laboratory examinations: $3-5 \mathrm{ml}$ of cubital venous blood was drawn before and after treatment, and the plasma was split and stored in a refrigerator at $-45^{\circ} \mathrm{C}$ after centrifugation. IL- 6 , IL- 8 , hs-CRP, TNF- $\alpha$, and ET were determined by enzyme-linked immunosorbent assay; $\mathrm{CD} 4+, \mathrm{CD} 8+$, and $\mathrm{CD} 4 / \mathrm{CD} 8$ ratio were tested using a Beckman Coulter DxFLEX flow cytometer. The levels of plasma D-lactic acid and DA0 were detected by enzymatic spectrophotometry; all the kits were purchased from Shanghai Enzyme-Linked Biotechnology Co., Ltd. and operated in strict accordance with the instructions.

QoL: The Inflammatory Bowel Disease Questionnaire (IBDQ) [15] was used before and after treatment for evaluating the QoL in patients between the two groups. The scale included 4 dimensions: emotional ability, social ability, intestinal and systemic symptoms, with a total of 32 items. Each item had a score of 1-7, with a full score of 32-224. The higher the score was, the higher the QoL of the patient was.

AEs: AEs such as abdominal discomfort during the treatment were recorded in detail.

\section{Statistical analysis}

The IBM Microsoft SPSS 21.0 software was used for statistical analysis of data. Measurement data was first tested for normality, and the data met the normal distribution was expressed as $\overline{\mathrm{x}} \pm \mathrm{s}$. Two independent sample $t$ test was used for comparisons between groups, and paired $t$ test was used for comparisons before and after treatment within the group; categorical data was expressed as rate (\%), and Chi-square $\chi^{2}$ test was used for comparison. $P<0.05$ was considered as statistically significant.

\section{Results}

Comparison of clinical efficacy between the two groups In the experimental group, the number of cases with cure, markedly effective, effective and invalid treatment was $35,17,8$, and 5 , respectively, with an effective rate of
92.31\%. The number of cases with cure, markedly effective, effective and invalid treatment in the control group was $21,12,17$, and 15 , respectively, with an effective rate of $76.92 \%$. The difference in effective rate between the two groups was statistically significant $\left(x^{2}=5.909\right.$, $P=0.015)$.

\section{Comparison of plasma inflammatory factors between the two groups}

The levels of plasma inflammatory factors between the two groups were comparable before treatment (all $P>0.05$ ). After treatment, the IL-6, IL-8, hs-CRP, and TNF- $\alpha$ of the two groups decreased, and were significantly lower in the experimental group than those in the control group (all $P<0.05$, Table 2);

\section{Comparison of $\mathrm{T}$ cell frequency between the two groups}

The levels of CD4+, CD8+, and CD4/CD8 were comparable between the two groups before treatment (all $P>0.05$ ). The levels of $\mathrm{CD} 4+$ and $\mathrm{CD} 4 / \mathrm{CD} 8$ ratio increased after treatment, and were significantly higher in the experimental group than those in the control group $(P<0.05$, Table 3$)$; the CD8+ levels were reduced, and were significantly lower in the experimental group than those in the control group $(P<0.05$, Table 3$)$;

\section{Comparisons of markers for intestinal mucosal barrier function between the two groups}

There was no significant difference in the plasma D-lactic acid, ET, and DA0 levels between the two groups before treatment (all $P>0.05$ ). After treatment, the plasma D-lactic acid, ET, and DA0 levels were decreased, and were lower in the experimental group than those in the control group (All $P<0.05$, Table 4);

\section{Comparisons of QoL between the two groups}

There was no significant difference in emotional ability, social ability, intestinal and systemic symptoms between the two groups before treatment (all $P>0.05$ ). After treatment, emotional ability, social ability, intestinal and systemic symptoms were improved in the two groups, and the scores were higher in the experimental group than those in the control group (all $P<0.05$, Table 5).

\section{Comparisons of AEs between the two groups}

During treatment, 2 cases of abdominal discomfort and 1 case of rash occurred in the experimental group, with an $\mathrm{AE}$ rate of $4.62 \%$ (3/65); 3 cases of abdominal discomfort and 2 cases of rash occurred in the control group, with an AE rate of $7.69 \%$ (5/65); there was no significant difference in the occurrence rate of AEs between the two groups $\left(\chi^{2}=0.533, P=0.718\right)$. 


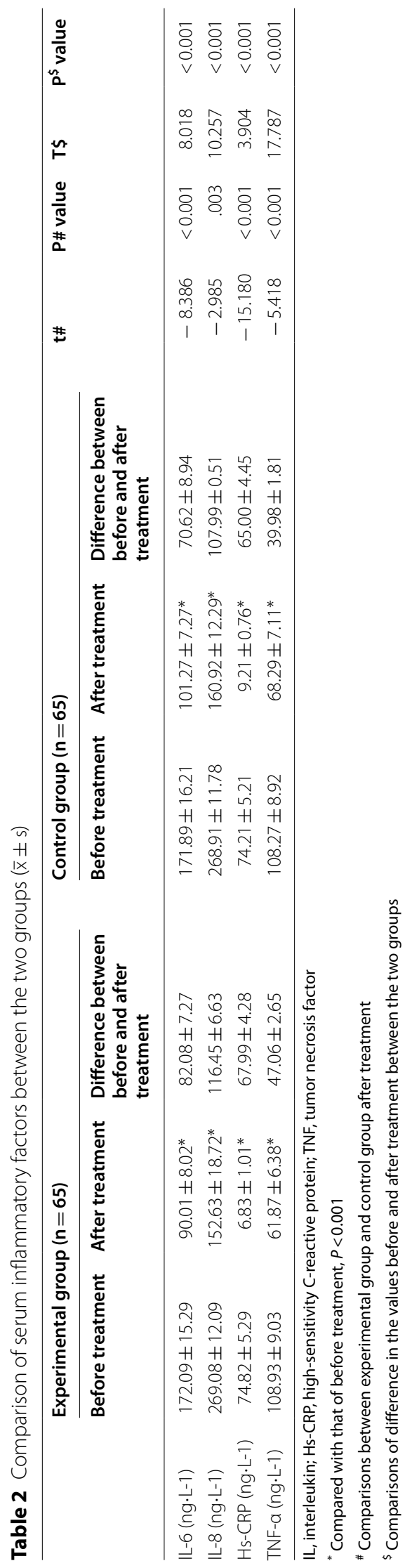




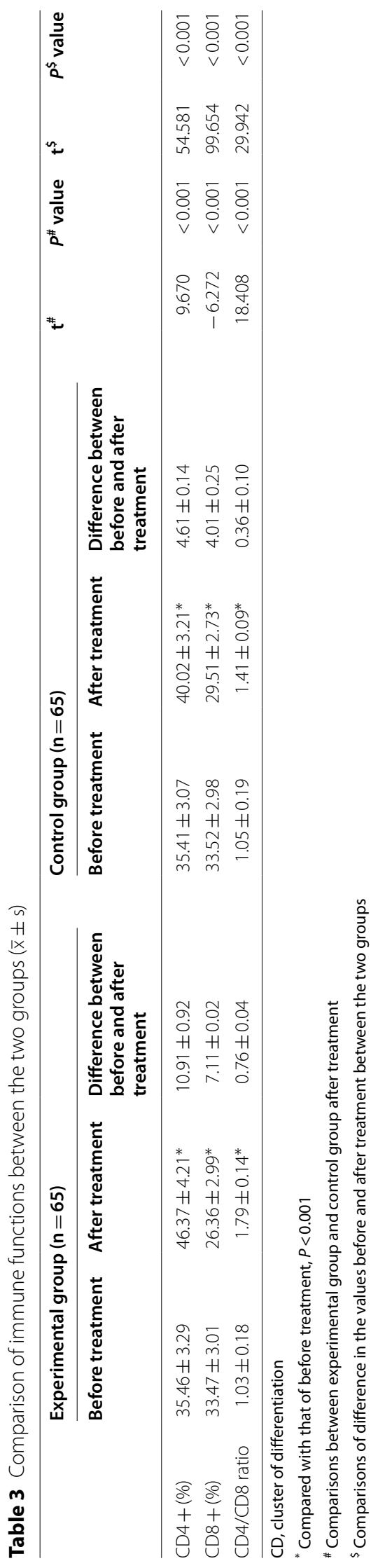




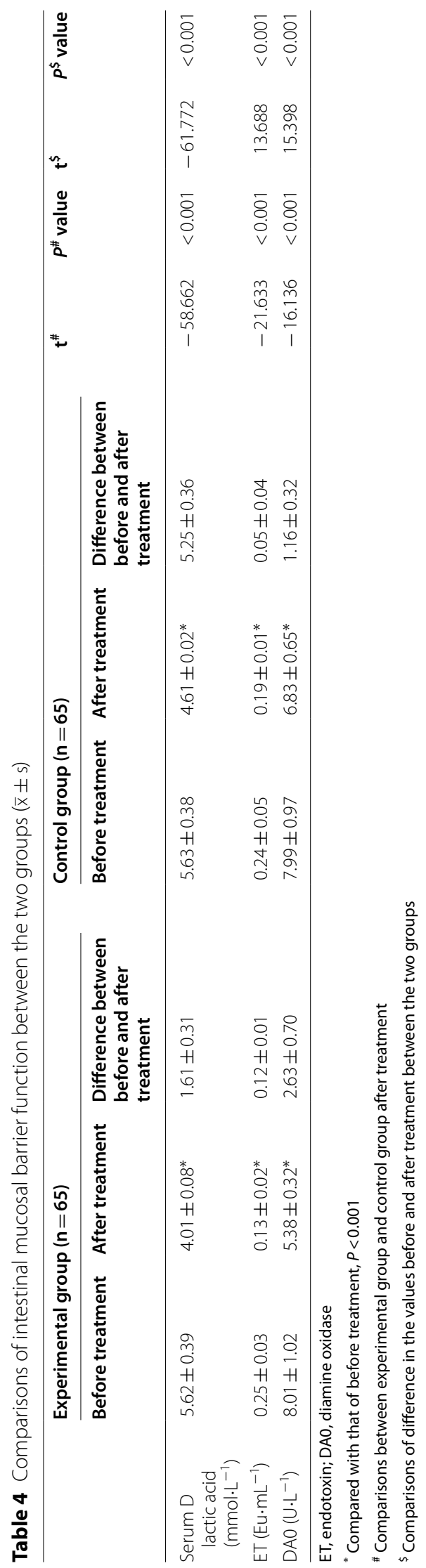




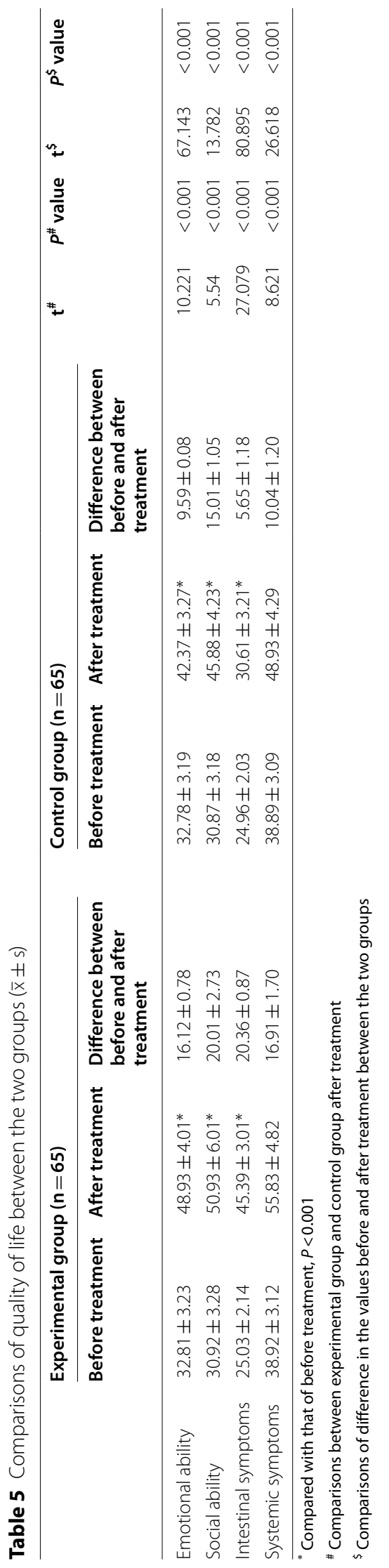




\section{Discussion}

Bifid triple viable capsule is a kind of probiotics composed of enterococcus, lactobacillus acidophilus and probiotic bifidobacterium, which can supplement the original intestinal flora, inhibit pathogenic bacteria, adhesion and forming a with intestinal mucosal epithelial cells. It has a protective effect on the intestinal mucosa, can prevent the invasion and colonization of pathogenic bacteria and opportunistic pathogens, regulate the imbalance of the intestinal flora, and improve clinical symptoms. Song Taoyan et al. [12] confirmed that mesalamine plus bifid triple viable capsules can effectively improve the clinical symptoms of ulcerative colitis patients. Zhang Jing et al. [10] showed that somatostatin plus mesalazine can significantly improve the intestinal function of ulcerative colitis patients. The results of this study showed that the clinical efficacy of the experimental group was higher than that of the control group $(P<0.05)$, suggesting that mesalamine plus somatostatin and bifid triple viable capsules can effectively improve the clinical symptoms of ulcerative colitis patients.

Inflammatory cytokines play an important role in the occurrence and development of ulcerative colitis [15]. The imbalance between anti-inflammatory and proinflammatory factors will accelerate the occurrence and progression of intestinal mucosal inflammation and is conducive to chronic development of inflammation [16]. IL-6, IL- 8 , hs-CRP, and TNF- $\alpha$ are all pro-inflammatory factors. IL- 6 plays an important role in the occurrence and development of ulcerative colitis, and its expression can reflect the degree of inflammation of ulcerative colitis [17]. IL-8 mediates pathological damage of colon mucosa and induces the inflammatory response of the intestinal mucosa of ulcerative colitis, which plays an important role in the pathogenesis of ulcerative colitis [18]. It can be used as an important indicator to evaluate the severity of ulcerative colitis [18]. Hs-CRP can reflect the severity and activity of ulcerative colitis patients [19]. TNF- $\alpha$ can promote the chemotaxis of chemoattract neutrophils, causing tissue cell infiltration, and inflammatory damage of the intestinal mucosa [20]. Therefore, this study evaluated plasma inflammatory factors in ulcerative colitis patients treated with mesalazine plus somatostatin and bifid triple viable capsules. The results showed that IL-6, IL-8, hs- CRP and TNF- $\alpha$ were lower in the experimental group than those in the control group $(P<0.05)$, suggesting that mesalazine plus somatostatin and bifid triple viable capsules can effectively reduce the plasma inflammatory factor levels in ulcerative colitis patients. Jiang Shengjun [21] confirmed that mesalazine and bifid triple viable capsules plus compound glutamine entericcoated capsules can improve the clinical symptoms of patients with mild to moderate ulcerative colitis during the active phase and reduce plasma inflammatory factors. Tan Yu'e [22] and colleagues have confirmed that the use of bifidobacteria and lactobacillus triple viable tablets plus olsalazine sodium capsules can reduce inflammatory factors in the treatment of ulcerative colitis patients. The mechanism may be due to the active probiotics of the bifidobacterium lactobacillus triple viable bacteria capsules [23], and the active probiotics can multiply in the intestines, increase the ratio of beneficial bacteria in the colorectal, inhibit the reproduction of pathogenic bacteria, and reduce the bacteria, improve the body's ability to absorb nutrients [24], thereby reducing the inflammatory response, and with the extension of time, the inflammatory response became increasingly lower [25].

The imbalance between the various subgroups of $\mathrm{T}$ cells will induce an immune response, as well as damage to the cells and aggravation of the inflammatory response [26]. The increase of CD8+ cells will enhance the function of CD4+ cells, promote activation of many B lymphocytes, activate humoral immunity, AND cause the production of a large number of immune complexes, thereby inducing mucosal congestion, edema, and ulcer formation [26]. The study by Shen Hao [26] confirmed that ulcerative colitis patients showed disorders of $\mathrm{T}$ cell subpopulations. Yu Haiping and colleagues [27] showed that the combination of bifid triple viable tablets plus mesalazine can improve the clinical efficacy and effectively improve the immune function of patients who were treated fro ulcerative colitis. The results of this study showed that the $\mathrm{CD} 4+$ and $\mathrm{CD} 4 / \mathrm{CD} 8$ ratios of the experimental group were higher than those of the control group, and the CD8+ level was lower than that of the control group $(P<0.05)$, suggesting that mesalazine plus somatostatin and bifidus triple capsules can effectively regulate $\mathrm{T}$ cell frequency of ulcerative colitis patients. Lu Lei et al. [28] confirmed that the adjuvant treatment of ulcerative colitis with bifid triple viable bacteria can regulate the levels of oxidative stress and inflammatory factors, inhibit the body's inflammatory response, and improve immune function. Studies by Tang Xuejun [25] and colleagues have confirmed that the adjuvant treatment of ulcerative colitis with bid triple viable powder can help inhibit the body's inflammatory response and improve immune function, and the effect was time dependent. The results of this study showed that after treatment, the CD4+ and $\mathrm{CD} 4 / \mathrm{CD} 8$ ratios of the experimental group were higher than those of the control group, and the CD8+ levels were lower than that of the control group $(P<0.05)$, suggesting that mesalazine plus somatostatin and bifid triple viable capsules can effectively regulate the immune function of ulcerative colitis patients. The mechanism may be due to the fact that the bifid triple viable capsule can increase the phagocytic ability of macrophages in 
the gastrointestinal tract, stimulate the body to secrete a large amount of IgA and IgG, prolong the lifespan of $\mathrm{T}$ lymphocytes, mediate phagocytosis, and maintain the balance of the intestinal flora, improve the immune function of the body, and with the extension of the time, the immune function will also increase [25].

The main pathological characteristics of patients with inflammatory bowel disease is the damage of intestinal mucosal barrier function [29], which is related to inflammatory response, mucosal immune function, intestinal mucosal permeability changes and intestinal mucosal membrane cell defects, which can accelerate intestinal mucosal cell apoptosis and increase intestinal endotoxins, or even induce intestinal failure [29]. Therefore, it is necessary to repair the intestinal mucosal barrier function of patients in the treatment of ulcerative colitis. Plasma D-lactic acid is a commonly used sensitive indicator that reflects the permeability of the intestine, and most of the intracellular enzymes exist in the small intestinal mucosa [30]. ET is an effective indicator of the damage of intestinal function and is composed of the cell wall of gram-negative bacteria [30]. DA0 is an effective indicator of the damage and repair of the intestinal barrier [30]. Most of the intracellular enzymes exist in the small intestinal mucosa. Zhao et al. [31] have confirmed that the plasma levels of D-lactic acid, ET, and DAO in ulcerative colitis patients were abnormally elevated and closely related to the severity of the disease. Feng Xianqing [32] confirmed that bifid triple viable capsules plus mesalazine can help improve the clinical efficacy of ulcerative colitis patients, which may be related to the improvement of immune function and the function of intestinal mucosal barrier of patients. The results of this study showed that the plasma D-lactic acid, ET and DAO of the experimental group were lower than those of the control group after treatment $(P<0.05)$, suggesting that mesalazine plus somatostatin and bifid triple viable capsules can effectively reduce the plasma D-lactic acid, ET, and DA0 levels of ulcerative colitis patients, thereby restoring the markers for intestinal mucosal barrier function. This may be because the bifid triple viable capsule can promote the repair and regeneration of intestinal epithelial tissue cells by producing butyric acid and acetic acid, forming a protective layer between the intestinal mucosa and microorganisms, and enhancing the barrier function of the intestinal mucosa [33].

Ulcerative colitis patients will be affected by psychology, society, and physiology during the development of the disease, which will affect the QoL of patients to varying degrees [34]. In recent years, with the transformation of the medical model to the physiological-psychological-social model, the QoL of patients has become a hot spot in clinical research, and it has also become one of the effective evaluation indicators after disease treatment [35]. Therefore, this study evaluated the QoL of ulcerative colitis patients after the treatment with mesalazine plus somatostatin and bifid triple viable capsules. The results showed that emotional ability, social ability, intestinal and systemic symptoms of patients in the experimental group were higher than those of the control group $(P<0.05)$, suggesting that mesalamine plus somatostatin and bifid triple viable capsules can effectively improve the QoL of ulcerative colitis patients. Wang Jia [36] confirmed that kangfuxin liquid plus bifid triple viable capsules can improve the QoL of ulcerative colitis patients, which is consistent with the results of this study. This study further analyzed the AEs and the results showed that the incidence of AEs in the two groups was comparable $(P>0.05)$, suggesting that mesalazine plus somatostatin and bifid triple viable capsules have relatively good safety and do not increase the AEs in ulcerative colitis patients.

\section{Limitations}

This study has some limitations. The sample size is small, and it is a single-center randomized controlled study. In future studies, a multi-center randomized controlled study with a large sample size should be conducted to further confirm the conclusions of this study. In addition, there is no placebo for the bifid triple viable capsules, which might affect the conclusions of this study. We will conduct clinical trials with placebo to further confirm these conclusions.

\section{Conclusion}

In conclusion, mesalazine plus somatostatin and bifid triple viable capsules can effectively improve the clinical symptoms of ulcerative colitis patients. It can reduce plasma inflammatory factors, regulate $\mathrm{T}$ cell frequency, restore markers for intestinal mucosal barrier function, and improve QoL, with high safety.

\section{Abbreviations \\ ET: Endotoxin; DA0: Diamine oxidase; IL-6: Interleukin-6; IL-8: Interleukin-8; hs- CRP: High-sensitivity C-reactive protein; TNF-a:Tumor necrosis factor.}

\section{Authors' contributions}

$Y L$ and $Y Z$ contributed to the conception and design of the study; $Y Y$ and DX performed the experiments, collected and analyzed data; SYL and YZ wrote the manuscript; All authors reviewed and approved the final version of the manuscript. All authors read and approved the final manuscript.

\section{Funding}

No funding was received for this study.

\section{Availability of data and material}

The datasets generated and analyzed during the current study are available from the corresponding author on reasonable request. 


\section{Declarations}

Ethics approval and consent to participate

The study protocol was approved by the Ethics Committee of Wuhan Emergency Medical Center. Informed consent was obtained from all the study subjects before enrollment.

\section{Consent for publication}

Not applicable.

\section{Competing interests}

The authors declare that they have no competing interests.

\section{Author details}

${ }^{1}$ General Department, The Sixth Hospital of Wuhan, Affiliated Hospital of Jianghan University, Wuhan, China. ${ }^{2}$ Department of Infection Management, The Sixth Hospital of Wuhan, Affiliated Hospital of Jianghan University, Wuhan, China. ${ }^{3}$ Department of Gastroenterology, The Sixth Hospital of Wuhan, Affiliated Hospital of Jianghan University, No.168 Hongkong Road, Wuhan 430015, China. ${ }^{4}$ Emergency Department, Wuhan Emergency Medical Center, No.288 Machang Road, Jianghan District, Wuhan 430022, China.

Received: 29 January 2021 Accepted: 27 July 2021

Published online: 04 August 2021

\section{References}

1. Keshteli AH, Madsen KL, Dieleman LA. Diet in the pathogenesis and management of ulcerative colitis; a review of randomized controlled dietary interventions. Nutrients. 2019;11(7):1498.

2. Tatiya-Aphiradee N, Chatuphonprasert W, Jarukamjorn K. Immune response and inflammatory pathway of ulcerative colitis. J Basic Clin Physiol Pharmacol. 2018;30(1):1-10.

3. Wang Y, Parker CE, Bhanji T, et al. Oral 5-aminosalicylicacid for induction of remission in ulcerative colitis. Cochrane Database Syst Rev. 2016,4(4):CD000543.

4. Liang SW, Wang XY, Qu CM, et al. Clinical study on changes of intestinal flora in patients with ulcerative colitis. J Med Res. 2015;44(9):60-2.

5. Ma PJ, Zhong JH, Cai W, et al. Effects of intestinal flora transplantation, probiotics combined with mesalazine on the intestinal barrier function of mild and moderate ulcerative colitis. Practical Hosp Clin J. 2020;17(06):84-7.

6. Jie Z. Observation and research on the effect of probiotics in the treatment of ulcerative colitis. China Pract Med. 2018;13(1):84-5.

7. Han GH, Wang CM. Research progress on the relationship between intestinal flora and ulcerative colitis. Harbin Med. 2017;03:88-9.

8. Zhang KQ, Jiang $\mathrm{Q}$, Zhang HB. Analysis of the effect of gastrointestinal function and intestinal flora in patients with ulcerative colitis after fecal bacteria transplantation. World Chin J Digest. 2019;027(001):68-72.

9. Shengjun J, Boneng M. Study on the treatment of mild to moderate ulcerative colitis during active period with mesalazine and bifidobacterium triple viable bacteria combined with compound glutamine. Hebei Med. 2018:40(23):21-5.

10. Jing Z, Jianyuan W, Meiqiao G, et al. Clinical study of somatostatin combined with mesalazine in the treatment of patients with ulcerative colitis. Chin J Clin Pharmacol. 2020;36(18): 2762-2764, 2768.

11. Jianming Q. Clinical application of octreotide in digestive system diseases. Mod J Integr Tradit Chin W Med. 2008;17(33):5235-7.

12. Taoyan S, Yongfeng Li. Clinical study of mesalazine combined with bifid triple viable bacteria capsules in the treatment of patients with ulcerative colitis. Chin J Clin Pharmacol. 2020;36(12):1610-3.

13. Inflammatory Bowel Disease Group, Digestive Disease Branch of Chinese Medical Association. Consensus opinions on the diagnosis and treatment of inflammatory bowel disease (2012 Guangzhou). Gastroenterology. 2012; 17(12): 763-81.

14. Inflammatory Bowel Disease Collaborative Group, Chinese Society of Digestive Diseases. Consensus opinions on the diagnosis and treatment of inflammatory bowel disease in my country. Chin J Digest. 2007;27(8): 545-50
15. Digestive System Disease Professional Committee of the Chinese Society of Integrated Traditional Chinese and Western Medicine. Guidelines for the Diagnosis and Treatment of Ulcerative Colitis Integrated Traditional Chinese and Western Medicine (Draft). Chin J Integr Tradit Chin W Med Digest. 2011;19(1): 61-5.

16. Anna $L$, Yuqing $L$, Muxiao $Z$, et al. Expression of inflammatory cytokines in patients with ulcerative colitis and its influence on prognosis. J S Med Univ. 2016;36(012): 1712-1717, 1731.

17. Qun X, Qing S, Xiancai Ge, et al. Expression and significance of IL- 6 and $\mathrm{IL}-23$ in the intestinal mucosa of patients with ulcerative colitis. Adv Mod Biomed. 2015;15(8):1471-3.

18. Yong Z. Analysis of the application value of detecting serum IL-1 $\beta$, IL- 6 and IL-8 levels in assessing the condition of patients with ulcerative colitis. Chin J Integr Tradit Chin W Med Digest. 2015;23(004):286-7.

19. Feng $H$, Ting $X$. The clinical value of serum I-FABP, $P C T$ combined with hs-CRP on ulcerative colitis. Cent S Med Sci J. 2018; 46(3): 305-307, 311

20. Rui Yi, Juan C, Xiaoyan L, et al. The expression and significance of TNF-a, IL-6 and IL-8 in the serum of patients with ulcerative colitis of different degrees. Adv Mod Biomed. 2015;15(014):2641-4.

21. Shengjun J, Boneng M. Study on the treatment of mild to moderate ulcerative colitis in active stage with mesalazine, bifidobacterium triple viable bacteria combined with compound glutamine. Hebei Med. 2018;40(23):3540-4.

22. Yu'e T, Donghong Y. Bifidobacterium lactobacillus triple viable tablet combined with olsalazine sodium capsule in the treatment of ulcerative colitis and its effect on the expression of IL-1 $\beta$ and IL-10. Mod J Integr Tradit Chin W Med. 2017;26(26):2898-900.

23. Pingfei $L$, Jian $B$. Changes of intestinal flora and serum inflammatory factor levels in patients with subclinical hepatic encephalopathy and the intervention effect of probiotics. Chin J Microecol. 2020;v.32(02):61-63+67

24. Xuejun T, Xiaoyun W, Gaojue W. Adjuvant treatment of ulcerative colitis with Bifidobacterium sanlianhuosan powder on inflammatory factors and T lymphocyte subsets. J Hainan Med Coll.2017;23(12):1620-1622, 1626.

25. Xuejun T, Xiaoyun W, Gaojue W. The effect of Bifidobacterium triple viable bacteria powder on inflammatory factors and $\mathrm{T}$ lymphocyte subsets in adjuvant treatment of ulcerative colitis. J Hainan Med Univ. 2017;23(12): 1620-1622, 1626.

26. Hao S. Clinical study of immune function status in patients with ulcerative colitis. Mod Digest Intervent Diagn Treat. 2016;21(4):554-6.

27. Haiping $Y$, Chungeng $L$. The effect of bifidobacteria triple viable tablets combined with mesalazine on immune function and intestinal mucosal barrier function in patients with ulcerative colitis. Mod J Integr Tradit Chin W Med. 2018;27(29): 3220-3223.

28. L Lei, $L$ Chuntao, Z Ye. The effect of the treatment of ulcerative colitis by triple live bifidobacterium on inflammatory factors, oxidative stress and T lymphocyte subsets. J Hainan Med Univ. 2017;23(16): 2192-2195, 2199.

29. Tao S, Jiangpeng W, Gang L, Mei H, Tong L. Observation of intestinal barrier function in patients with ulcerative colitis. Chin Med J. 2015;95(24):1941-3.

30. Chunlong D, Yuhai W, Likun Y, et al. The role of serum D-lactic acid, bacterial endotoxin and diamine oxidase in the evaluation of intestinal mucosal barrier function after traumatic brain injury. Chin J Neurosurg. 2019;035(012):1258-61.

31. Zhao $L$, Wu H, Zhao A, et al. The in vivo and in vitro study of polysaccharides from a two-herb formula onulcerative colitis and potential mechanism of action. J Ethnopharmacol. 2014;153(1):151-9.

32. Xianqing $F$, Chuan $L$, Ran $L$, et al. Effects of Bifidobacterium triple viable capsule combined with mesalazine on immune function and intestinal mucosal barrier function in patients with ulcerative colitis. Int J Digest Dis. 2018;38(02):69-72.

33. Xiaodong D, Lifei L. Changes of intestinal flora and intestinal mucosal barrier in patients with ulcerative colitis and the intervention effect of probiotics. Chin J Microecol. 2019;31(02):75-8.

34. Zhijuan W, Yaling J, Chunxia S. The effect of psychological nursing on anxiety, depression and quality of life in patients with chronic ulcerative colitis. Chin J Mod Nurs. 2016;22(15):2159-62. 
35. Junxia L, Tingting G, Huahong W, Xinguang L. Quality of life characteristics and influencing factors of patients with ulcerative colitis in remission. Chin J Pract Intern Med. 2013;33(12):961-4.

36. Jia W, Aixia C, Hongchao Li. The effect of Kangfuxin liquid combined with Bifidobacterium triple viable capsules on serum LPO, SOD levels and quality of life in patients with ulcerative colitis. China J Mod Med. 2019;29(15):82-6

\section{Publisher's Note}

Springer Nature remains neutral with regard to jurisdictional claims in published maps and institutional affiliations.
Ready to submit your research? Choose BMC and benefit from:

- fast, convenient online submission

- thorough peer review by experienced researchers in your field

- rapid publication on acceptance

- support for research data, including large and complex data types

- gold Open Access which fosters wider collaboration and increased citations

- maximum visibility for your research: over 100M website views per year

At BMC, research is always in progress.

Learn more biomedcentral.com/submissions 